EPJ Web of Conferences 57, 01001 (2013)

DOI: $10.1051 /$ epjconf/20135701001

(C) Owned by the authors, published by EDP Sciences, 2013

\title{
Ultracold fermions with repulsive interactions
}

\author{
W. Ketterle
}

MIT, Cambridge, MA 02139, USA

\begin{abstract}
An ultracold Fermi gas with repulsive interaction has been studied. For weak interactions, the atomic gas is metastable, and the interactions were characterized by obtaining the isothermal compressibility from atomic density profiles. For stronger interactions $\left(\mathrm{k}_{\mathrm{F}} \mathrm{a} \approx 1\right)$, rapid conversion into Feshbach molecules is observed. When the conversion rate becomes comparable to the Fermi energy divided by $\eta$, the atomic gas cannot reach equilibrium without forming pairs. This precludes the predicted transition to a ferromagnetic state (Stoner transition). The absence of spin fluctuations proves that the gas stays paramagnetic. In free space, a Fermi gas with strong short-range repulsion does not exist because of the rapid coupling to molecular states.
\end{abstract}

\section{INTRODUCTION}

Many-body systems can often be modeled using contact interactions, greatly simplifying the analysis while maintaining the essence of the phenomenon to be studied. Such models are almost exactly realized with ultracold gases due to the large ratio of the de Broglie wavelength to the range of the interatomic forces $[1,2]$.

For ultracold fermions with mass $\mathrm{m}$ and contact interactions $\mathrm{U}_{0} \delta\left(\mathrm{r}-\mathrm{r}^{\prime}\right)$, the Hamiltonian $\mathrm{H}$ is

$$
H=\sum_{k}\left(\frac{\hbar^{2} k^{2}}{2 m}\right)\left(a_{k, \uparrow} a_{k, \uparrow}^{\dagger}+a_{k, \downarrow} a_{k, \downarrow}^{\dagger}\right)+U_{0} \sum_{k, l, q} a_{k-q, \downarrow}^{\dagger} a_{l+q, \uparrow}^{\dagger} a_{k, \downarrow} a_{l, \uparrow} .
$$

The first term is the kinetic energy, the second the interaction term. Due to the Pauli principle, there is no short range interaction between atoms in the same spin state. The interaction parameter $\mathrm{U}_{0}$ is proportional to the scattering length a: $\mathrm{U}_{0}=4 \pi \hbar^{2} \mathrm{a} / \mathrm{m}$, where $\mathrm{m}$ is the atomic mass. For negative scattering length, this Hamiltonian describes pairing of fermions into Cooper pairs and BCS superfluidity [1]. For positive scattering length the BEC limit is realized where the fermions form composite bosons with a binding energy of $\hbar^{2} / \mathrm{ma}^{2}$. The ground state is a Bose-Einstein condensate of atom pairs.

The existence of a bound state for positive scattering length and short range interactions follows from the expression for the s-wave scattering amplitude $\mathrm{f}(\mathrm{k})=1 /(-1 / \mathrm{a}-\mathrm{ik})$ which has a pole on the imaginary axis at $\mathrm{k}=\mathrm{i} / \mathrm{a}$, corresponding to a bound state of binding energy $\hbar^{2} / \mathrm{ma}^{2}$. More generally, if the potential is not a delta function potential, the low energy expansion of the scattering amplitude involves the effective range $\mathrm{r}_{e}$ and reads $\mathrm{f}(\mathrm{k})=1 /\left(-1 / \mathrm{a}+\mathrm{r}_{e} \mathrm{k}^{2} / 2-\mathrm{ik}\right)$. The absence of a bound state, i.e. no pole on the imaginary axis, requires $r_{e}>a / 2$, i.e. an interaction potential which is not short range any more.

The Hamiltonian in Eq. (1) is frequently used to describe Fermi gases with short range repulsive interactions. However, the discussion above emphasizes that this neglects the presence of at least one bound state which has to be present due to fundamental properties of the scattering amplitude.

This is an Open Access article distributed under the terms of the Creative Commons Attribution License 2.0, which permits unrestricted use, distribution, and reproduction in any medium, provided the original work is properly cited. 


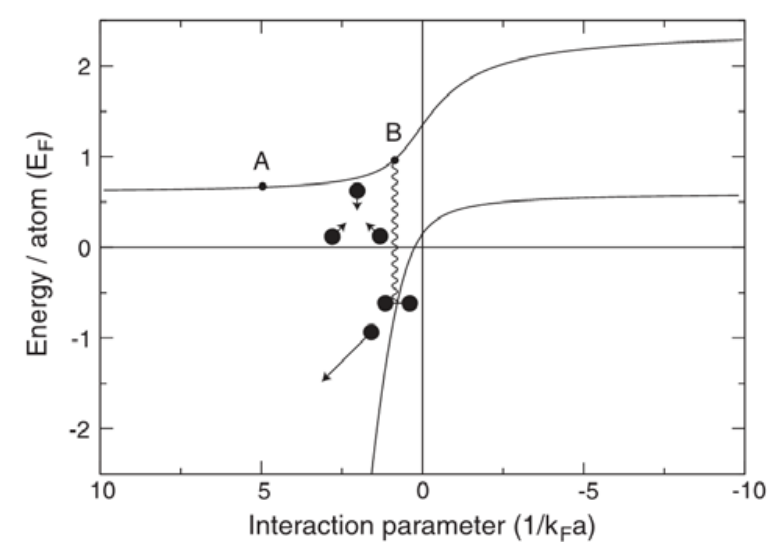

Figure 1. Diagram showing relevant energy levels for a Fermi gas with short-range interactions. The interactions are described by the scattering length a. For negative scattering length, the branch with attractive interactions is the lowest state and therefore (meta-)stable. For positive scattering length, the upper (repulsive) branch is connected to the lower (paired) branch by 3-body collisions. Adapted from Refs. [10, 11].

A natural question is then to what extent can we prepare and study an atomic system with short range repulsive interactions and neglect the bound pair state? The time scale for conversion into pairs is given by the rate of three-body recombination. If this rate is slow compared to equilibration rates, then the system stays on the repulsive branch of the energy diagram in Figure 1 and we can speak of an atomic gas with repulsive interactions. For bosonic gases, this is the regime in which weakly interacting BoseEinstein condensates have been studied. If the conversion to pairs is fast then it becomes impossible to study a gas with repulsive interactions. Such a gas would represent a region of Hilbert space which is so strongly coupled to other parts (involving bound pairs) that one cannot look at this region in isolation, but has to include bound pairs in the description. Any theoretical description which projects only onto the unbound states $[3,4]$ is internally inconsistent and does not describe a system which can be realized in Nature (but those simulations are useful to understand individual mechanisms separately).

We present here experimental work studying repulsive interactions and pair formation in Fermi gases [5-7]. We show that for small scattering length recombination into pairs is slow, and one can study the properties of the atomic system in equilibrium. For large scattering length, the recombination rate becomes comparable to the Femi energy $\mathrm{E}_{\mathrm{F}}$ (divided by $\hbar$ ) and the gas will convert into pairs before equilibrating. It is in this regime where the simplest model for itinerant ferromagnetism has been formulated, the so-called Stoner model [8,9]. Our work shows that the Stoner model, at least in its basic form, cannot be realized in Nature due to rapid pair formation.

\section{DETERMINATION OF THE COMPRESSIBILITY}

The compressibility is a key quantity characterizing a many-body system. Repulsive interactions reduce the compressibility of a gas, attractive interactions increase it. The compressibility can be directly obtained from density profiles in a harmonic trapping potential [7]. In essence, the compressibility is the (normalized) curvature of the atomic density in equilibrium in the trapping potential.

The determination of the compressibility (as the determination of the equation of state [12]) requires fully equilibrated density profiles, and therefore a lifetime of the cloud against inelastic collisions which is comparable to the trap period. We characterized the lifetime of the cloud by quickly ramping the magnetic field close to the broad Feshbach resonance at 832 Gauss and monitoring the decay of the atom number and the increase in the molecular fraction (Figure 2). As expected we find only a limited 


\section{ICAP 2012}
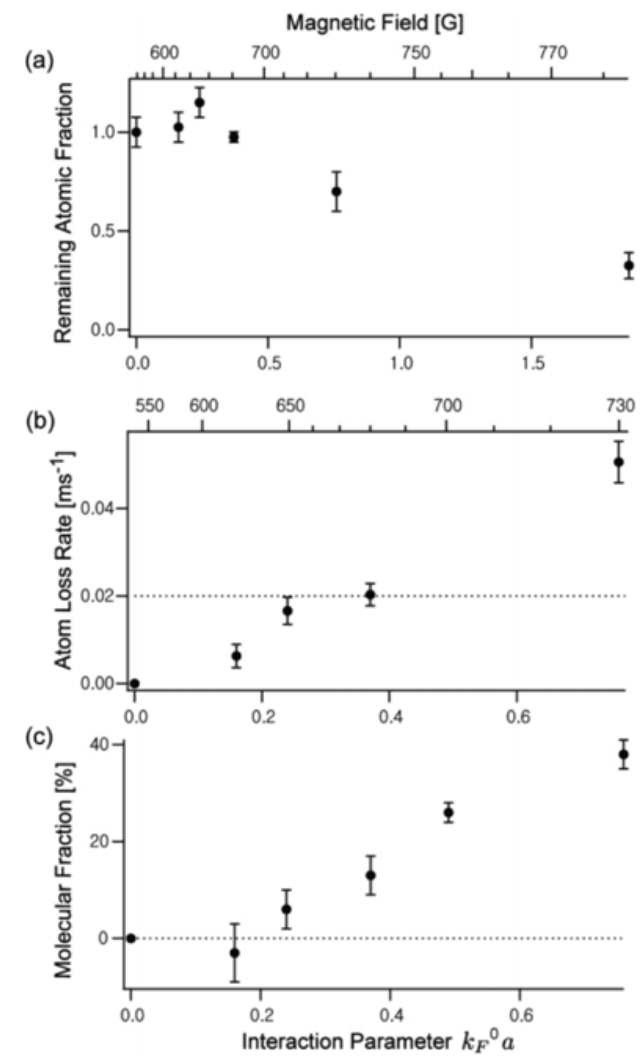

Figure 2. Characterizing atomic loss for increasing repulsive interactions. (a) Remaining fraction of atom number immediately after the fastest possible ramp to the target field. (b) Atom loss rates at the target fields. Dashed line shows the estimated maximum tolerable loss rate for equilibration of $0.02 / \mathrm{ms}$. (c) Molecular fraction after the $50 \mathrm{~ms}$ ramp and $30 \mathrm{~ms}$ wait time, corresponding to when we measure equilibrium atomic density profiles. Figure from Ref. [7].

window for metastability where we can observe equilibrated clouds. This window limits the interaction strength to $\mathrm{k}_{\mathrm{F}} \mathrm{a}<0.35$.

An analysis of the density profiles provides the compressibility as a function of $\mathrm{k}_{\mathrm{F}} \mathrm{a}$ (Figure 3 ). It agrees well with first-order perturbation theory at weak interactions. This is the first observation of the mean-field term in a thermodynamic quantity for repulsively interacting fermions. For stronger interaction, the atoms rapidly convert into pairs making it impossible to characterize the equilibrium density distribution of the atomic gas. The signal-to-noise ratio (and some heating) prevented us from discerning the second-order interaction term which is the Lee-Huang-Yang correction for fermions.

\section{RAPID PAIR FORMATION IN A REPULSIVELY INTERACTING FERMI GAS}

In a second experiment (actually carried out before the experiment described above) we have probed much shorter time scales by jumping the magnetic field close to the Feshbach resonance using the discharge of a high-voltage capacitor [11].

When we approached the Feshbach resonance, we found a rapid decay into molecules which was faster than our time resolution (Figure 4). About $30 \%$ of atom loss occurs already during the relevant $100 \mu \mathrm{s}$ of ramping through the strongly interacting region, indicating a lower bound of around $310^{3} \mathrm{~s}^{-1}$ 


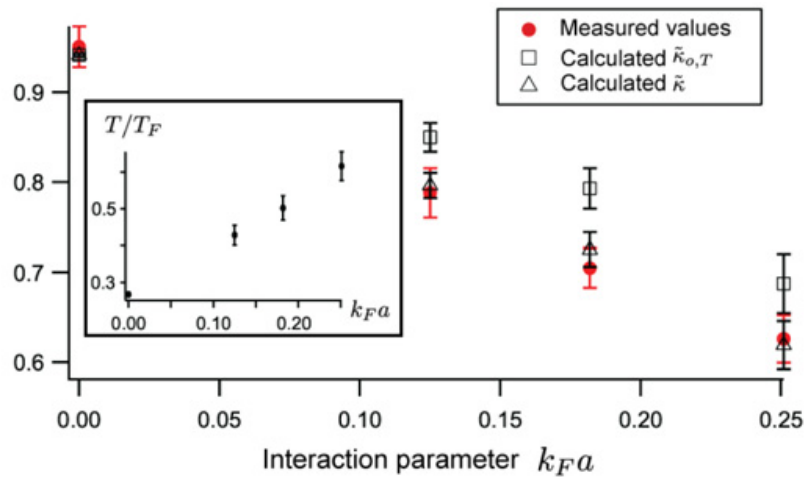

Figure 3. Measured isothermal compressibility at various interaction strengths. The compressibility is normalized by the value for a non-interacting gas. Open squares show the calculated compressibility of a non-interacting gas at the measured temperature. The compressibility (red circles) decreases due to repulsive interaction as well as finite temperature. These two effects must be separated, and hence the interaction effect is the difference between the open squares and the red circles. Open triangles show the calculated compressibility using first-order perturbation theory, which agrees with the measured compressibility. The temperature of the gas increases with interaction strength due to increasing rates of pair formation (inset). Figure adapted from Ref. [7].

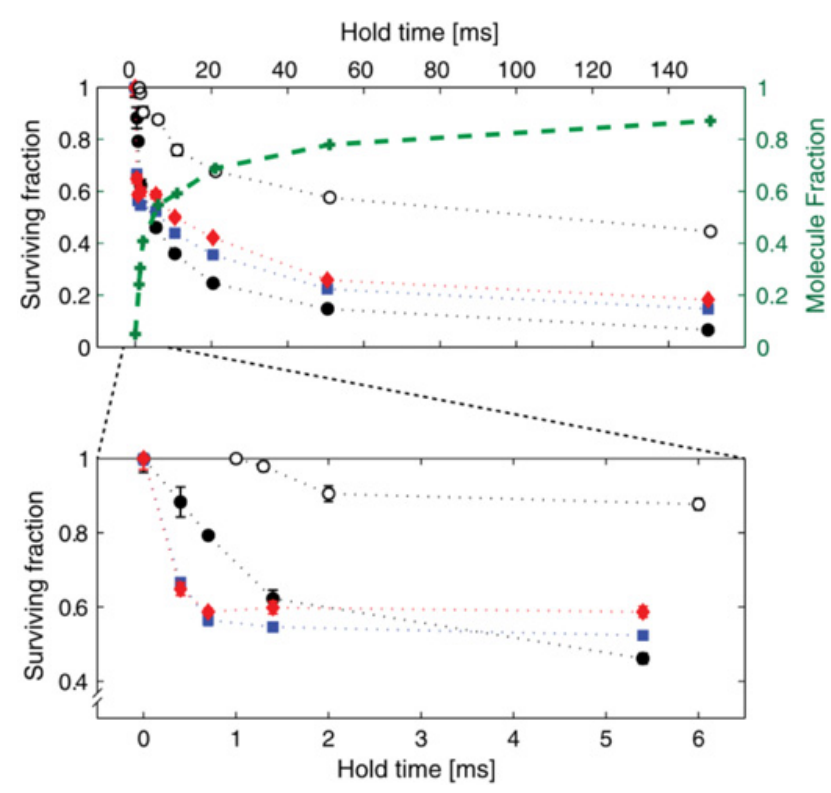

Figure 4. Characterization of molecule formation at short and long hold times, and at different values of the interaction strength. The closed symbols, circles (black) at $790 \mathrm{G}$ with $\mathrm{k}_{\mathrm{F}} \mathrm{a}=1.14$, squares (blue) at $810 \mathrm{G}$ with $\mathrm{k}_{\mathrm{F}} \mathrm{a}=2.27$ and diamonds (red) at $818 \mathrm{G}$ with $\mathrm{k}_{\mathrm{F}} \mathrm{a}=3.5$ represent the normalized number of free atoms, the open symbols the total number of atoms including those bound in Feshbach molecules (open circles at $790 \mathrm{G}$ with $\mathrm{k}_{\mathrm{F}} \mathrm{a}=1.14$ ). The crosses (green) show the molecule fraction.

for the loss rate which is $13 \%$ of the inverse Fermi time $\mathrm{E}_{F} / \hbar$ calculated with a cloud averaged Fermi energy. Since the Fermi time is the shortest possible time scale for local equilibration, it is clear that the rapid conversion into pairs prevents the atomic gas from reaching any metastable equilibrium state. 


\section{ABSENCE OF FERROMAGNETISM}

For itinerant fermions with strong short-range repulsion, textbook calculations (based on mean-field approximation or second order corrections) predict a ferromagnetic phase transition, the so-called Stoner model $[8,9]$. Intuitively, when repulsive interactions become too strong, the paramagnetic gas phase separates into a spin up and spin down domain. Such a ferromagnetic state has no interaction energy (since spin up and down atoms are spatially separated), but a kinetic energy (Fermi energy) which is increased by a factor of $2^{2 / 3}$ [5]. A ferromagnetic phase transition is approached with a divergent magnetic susceptibility [13]. According to the fluctuation dissipation theorem, local spin fluctuations are proportional to the susceptibility.

We monitor spin fluctuations by observing a speckle pattern after a detuned laser beam has traversed the cloud [11]. The spin fluctuations lead to index of refraction fluctuations which imprint a speckle pattern into the transmitted beam. Compared to an ideal Fermi gas, the variance of the spin fluctuations should increase by the number of atoms per domain, and diverge at the ferromagnetic phase transition. Since our method can resolve the spin fluctuations of an ideal Fermi gas [14], it is suitable to observe the onset of domain formation with domains as small as just a few atoms.

For various strengths of the interactions and various hold times, we observed the absence of any major increase of spin fluctuations. This shows conclusively that the samples remain in the paramagnetic phase for arbitrarily large scattering length [11] and does not form any ferromagnetic domains, even in a transient way [11].

A previous experiment in our group [3] reported evidence for ferromagnetism by presenting nonmonotonic behavior of atom loss rate, kinetic energy and cloud size when approaching the Feshbach resonance, in agreement with predictions based on the Stoner model. Our measurements confirm that the properties of the gas strongly change near $\mathrm{k}_{\mathrm{F}} \mathrm{a} \approx 1$. However, the more detailed study rules out that Ref. [3] has observed ferromagnetic behavior.

Our conclusion is that an ultracold gas with strong short range repulsive interactions near a Feshbach resonance remains in the paramagnetic phase. The fast formation of molecules and the accompanying heating makes it impossible to study such a gas in equilibrium, confirming predictions of a rapid conversion of the atomic gas to pairs $[15,16]$. The Stoner criterion for ferromagnetism applies when the effective interaction strength times the density of states is larger than one. This is at least an approximately valid criterion for multi-band lattice models [17]. We have shown here that this criterion cannot be applied to Fermi gases with short-range repulsive interactions (the basic Stoner model) since the neglected competition with pairing is crucial. It may be possible to suppress the pair formation in optical lattices or in lower dimensions.

The case of the Fermi gas with repulsive interactions illustrates the capability to simulate Hamiltonians using clean systems of ultracold atoms [12]. For strong interactions, the simulations approach parameter regimes for which controlled calculations are no longer possible. In the case above, we could show that a phase transition predicted by state-of-the-art calculations, does not take place.

This work was supported by the NSF and ONR, an AFOSR MURI, and by ARO grant no. W911NF-07-1-0493 with funds from the DARPA Optical Lattice Emulator program.

\section{References}

[1] W. Ketterle and M.W. Zwierlein, in Ultracold Fermi Gases, Proceedings of the International School of Physics "Enrico Fermi”, Course CLXIV, Varenna, 20-30 June 2006, edited by M. Inguscio, W. Ketterle, and C. Salomon (IOS Press,, Amsterdam, 2008) p. 95.

[2] I. Bloch, J. Dalibard, and W. Zwerger, Rev. Mod. Phys. 80, 885 (2008).

[3] S. Pilati, G. Bertaina, S. Giorgini, and M. Troyer, Phys. Rev. Lett. 105, 030405 (2010). 
[4] S.-Y. Chang, M. Randeria, and N. Trivedi, Proc. Natl. Acad. Sci. USA 108, 51 (2011).

[5] G.-B. Jo, Y.-R. Lee, J.-H. Choi, C.A. Christensen, T.H. Kim, J.H. Thywissen, D.E. Pritchard, and W. Ketterle, Science 325, 1521 (2009).

[6] C. Sanner, E.J. Su, A. Keshet, W. Huang, J. Gillen, R. Gommers, and W. Ketterle, Phys. Rev. Lett. 106, 010402 (2011).

[7] Y.-R. Lee, M.-S. Heo, J.-H. Choi, T.T. Wang, C.A. Christensen, T.M. Rvachov, and W. Ketterle, Phys. Rev. A 85, 063615 (2012).

[8] D.W. Snoke, Solid State Physics: Essential Concepts (Addison-Wesley, San Francisco, 2008).

[9] E.C. Stoner, Phil. Mag. 15, 1018 (1933).

[10] L. Pricoupenko and Y. Castin, Phys. Rev. A 69, 051601 (2004).

[11] C. Sanner, E.J. Su, W. Huang, A. Keshet, J. Gillen, and W. Ketterle, Phys. Rev. Lett. 108, 240404 (2012).

[12] I. Bloch, J. Dalibard, and S. Nascimbène, Nature Physics 9, 267 (2012).

[13] A. Recati and S. Stringari, Phys. Rev. Lett. 106, 080402 (2010).

[14] C. Sanner, E.J. Su, A. Keshet, R. Gommers, Y.-I. Shin, W. Huang, and W. Ketterle, Phys. Rev. Lett. 105, 040402 (2010).

[15] D. Pekker, M. Babadi, R. Sensarma, N. Zinner, L. Pollet, M.W. Zwierlein, and E. Demler, Phys. Rev. Lett. 106, 050402 (2011).

[16] S. Zhang and T.L. Ho, New J. Phys. 13, 055003 (2011).

[17] J. Bünemann, W. Weber, and F. Gebhard, Phys. Rev. B 57, 6896 (1998). 